\title{
Effect of gallic acid on liver injury during obstructive cholestasis after bile duct ligation in rat
}

\author{
Erol Basuguy ${ }^{1} \cdot$ Mehmet Hanifi Okur $^{1} \cdot$ Serkan Arslan $^{1} \cdot$ Hikmet Zeytun $^{1} \cdot$ Gulay Aydogdu $^{2}$. \\ Aysun Ekinci ${ }^{3}$ \\ Department of Pediatric Surgery, Pathology ${ }^{2}$ and Biochemistry, Faculty of Medicine, Dicle University, Diyarbakir, \\ Turkey
}

\section{ABSTRACT}

\begin{abstract}
Aim: To investigate the hepatoprotective, anti-inflammatory and antioxidant effects of gallic acid (GA) against obstructive cholestasis (OC) -induced liver damage in rats.

Methods: Thirty female Sprague-Dawley rats were divided into three groups. Group $1(\mathrm{n}=10)$ was the shamoperated group. In group 2 and group 3, hepatoduodenal ligament dissection was performed after laparotomy. Once the common bile duct was made apparent, it was ligated with 4/0 silk surgical suture and cut between both sutures. Group 2 ( $\mathrm{n}=10)$ was the control group. Group $3(\mathrm{n}=10)$ was the GA group. GA $(50 \mathrm{mg} / \mathrm{kg})$ was administered by oral gavage daily for 10 days. At the end of the experiment on day 10 , the rats were anesthetized. Fibrosis, inflammation, ductal proliferation and necrosis were evaluated histopathologically. Serum levels of AST, ALT, TBIL, DBIL, LDH and GGT levels were determined. In the serum and liver, TAS, TOS, MDA, TNF- $\alpha$, IL-1, IL-6, and IL 10 levels were evaluated.

Results: When group 2 and group 3 were compared histopathologically, fibrosis and inflammation were significantly lower in group 3. In group 3, all LFTs (except DBIL), liver and serum IL-6, IL-1, TOS, MDA, and TNF- $\alpha$ levels were significantly lower than group 2, whereas IL-10 and TAS values were increased.

Conclusion: Findings of this research indicate that GA may be effective against OC-induced liver damage in a rat model. We presume that the beneficial effects of GA are closely associated with its antioxidant and antiinflammatory activities. Therefore, we think that using GA can save us time before resorting to the surgical method.
\end{abstract}

Keywords: Obstructive cholestasis, bile ducts ligation, rats, gallic acid, liver, oxidative stress, fibrosis.

$\begin{array}{ll}\square \text { Dr. Erol Basuguy, } & \begin{array}{l}\text { congenital bile duct anomalies, infectious } \\ \text { Department of Pediatric Surgery, Dicle University, } \\ \text { Faculty of Medicine, Diyarbakir, Turkey }\end{array} \\ \begin{array}{l}\text { E-mail: erbas.80@hotmail.com } \\ \text { Received: 2020-10-14/Revised: 2020-10-20 }\end{array} & \begin{array}{l}\text { primary sclerosing cholangitis, abdominal } \\ \text { traumas, and cholangitis associated with }\end{array} \\ \text { Accepted: 2020-11-19/Published online: 2021-01-01 } & \begin{array}{l}\text { Langerhans cell histiocytosis [1,2]. } \\ \text { The liver is the primary organ affected by OC. }\end{array} \\ \text { Introduction } & \begin{array}{l}\text { Retention of hydrophobic bile salts via } \\ \text { membranes causes it to accumulate in }\end{array} \\ \text { Obstructive cholestasis (OC) is a common } & \begin{array}{l}\text { hepatocytes, thereby disrupting membrane } \\ \text { condition that requires surgery. }\end{array} \\ \begin{array}{l}\text { Choledocholithiasis is the most common cause } \\ \text { of OC; other causes include biliary cyst }\end{array} & \begin{array}{l}\text { fluidity and function and causing cell damage } \\ \text { stenosis, pancreatitis, parasitic infections, }\end{array}\end{array}$


several organs and physiological processes. Bilirubin accumulates at high levels in patients with $\mathrm{OC}$ due to dysfunctional bile secretion. Thus, bile acid in hepatocytes causes acute liver damage as a result of oxidative stress [4]. Subsequently, hemostatic abnormalities, cardiac disorders, and multiple system disorders (e.g., renal failure) occur, which cause changes in patient immunity [5]. It remains unknown why OC causes multiple organ dysfunction; however, bilirubin breakdown and impaired bile acid homeostasis have been suggested [6].

Although the most common treatment for OC is surgery, percutaneous transhepatic biliary drainage and medical treatment are occasionally needed to stabilize the patient's condition and restore biliary flow before surgery [7]. Therefore, drugs are primarily used to reduce hepatic damage caused by inflammatory cells and elevated bilirubin levels, as well as to reduce elevated biliary pressure in ducts and portal veins. For prevention of intrahepatic cholestasis, novel drugs are needed to provide an alternative to surgical treatment of OC [8].

In numerous scientific reports, polyphenolic compounds were demonstrated to have different pharmacological activities that may be associated with the antioxidant system [9]. In in vitro studies [10], the ethyl acetate fraction (EF) was shown to have an antifibrotic effect. Based on mass spectrometry, EF has two main components: gallic acid (GA) and ellagic acid [11]. Gallic acid is a natural phenolic antioxidant; it is found in green tea leaves, sumac, gallnuts, witch hazel, mulberries, blackberries, oak bark, raspberries, strawberries, and dragon fruit [12]. The antioxidant effects of GA are presumably due to the hepatoprotective activity of hydroxyl groups [13]. GA has attracted considerable attention due to its antioxidant effects and the cleaning properties of reactive oxygen species (ROS) $[14,15]$.

In this study, the hepatoprotective effects of gallic acid (GA) against liver damage in obstructive cholestasis (OC) model caused by bile duct ligation in rats were investigated.

\section{Materials and Methods}

\section{Animals}

Thirty female Sprague-Dawley rats (230-270

g) were obtained from Dicle University Animal Experimental Care Unit (Diyarbakir, Turkey). All experiments were approved by the Dicle University Animal Experiments Local Ethics Committee (DUHADEK) (Decision No: 14TF-149) and were performed in compliance with the DUHADEK guidelines. Rats were housed in cages and fed with rat diet and tap water ad libitum at a room temperature of $26 \pm 3 \circ \mathrm{C}$, a relative humidity of $\% 55-70$, and a time period of $12 \mathrm{~h}$ light and $12 \mathrm{~h}$ dark.

\section{Groups and surgical method}

Animals were divided into three groups. Each group was consisted of ten animals. After an overnight fasting, rats were anesthetized before the experimental procedure. The rats' abdomens were shaved and cleaned with $10 \%$ povidone-iodine solution. Under sterile conditions, an incision was made at the midline of the abdomen (i.e., laparotomy was performed); the liver, diaphragm, and adjacent organs with apparent hepatoduodenal ligament were carefully dissected.

Group $1(\boldsymbol{n}=10)$ was the sham group. After laparotomy, the hepatoduodenal ligament was dissected; the common bile duct was exposed, but no binding or cutting was conducted. Then, the animals were given standard feed and water for 10 days.

Group $2(n=10)$ was the control group. After laparotomy, hepatoduodenal ligament 
dissection was performed. Once the common bile duct was made apparent, then it was ligated with $4 / 0$ silk surgical suture and cut between both sutures [16]. Animals were given standard feed and water for 10 days.

Group $3(\boldsymbol{n}=10)$ was the GA group, which underwent the same procedures as in group 2 . In addition, $50 \mathrm{mg} / \mathrm{kg}$ GA dissolved in $1 \mathrm{~mL}$ saline solution (Gallic acid ${ }^{\circledR}, 97.5-102.5 \%$ (titration), Sigma-Aldrich, Shanghai, China) was administered by oral gavage daily for 10 days [17].

After surgical procedures had been completed, the abdominal incisions were sutured en bloc with $3 / 0$ silk sutures. Postoperative feeding was allowed. The rats were kept in the preoperative room and fed as described above. On the $10^{\text {th }}$ day, the rats were anesthetized. Then, an abdominal incision was made, liver samples were collected, and blood was drawn. Finally, the animals were sacrificed by exsanguination. Blood samples were centrifuged at $4^{\circ} \mathrm{C}$ and 4,000 rpm for $15 \mathrm{~min}$. Plasma samples $(200 \mu \mathrm{L})$ were obtained and stored at $-80^{\circ} \mathrm{C}$ for biochemical parameter analysis.

Two rats (one in Group 2 and one in Group 3) died during the course of the experiment, prior to sample collection, and that replaced with new ones.

\section{Histopathological examination}

Dissected liver tissues were processed in $10 \%$ formalin solution, then embedded in paraffin wax in accordance with a routine embedding protocol. Next, 4- $\mu$ m-thick sections were cut from paraffin blocks. In addition to hematoxylin and eosin staining, Masson's trichrome (Bioptica, Milan, Italy) staining was performed on liver slides to facilitate the evaluation of periportal fibrotic bands. Sections were evaluated using a Zeiss imager A2 microscope. Fibrosis, inflammation, and necrosis were evaluated histopathologically in accordance with the method described by Ishak et al. [18], ductal proliferation was evaluated in accordance with the method described by Ara et al. [19]

\section{Liver function tests (LFTs)}

For LFTs, sera were obtained by centrifuging blood samples at $4,000 \mathrm{~g}$ for $5 \mathrm{~min}$. Subsequently, an automated biochemical analyzer (Abbott Laboratories, Chicago, IL, USA) was used, in accordance with the manufacturer's protocol, to determine the levels of aspartate aminotransferase (AST), alanine aminotransferase (ALT), direct bilirubin (DB), total bilirubin (TB), lactate dehydrogenase (LDH) and gamma-glutamyl transferase (GGT).

Assessment of oxidative stress and inflammatory cytokines in liver and serum

The total oxidant status (TOS) and total antioxidant status (TAS) in serum samples were determined using commercial kits (Rel Assay Diagnostics, Gaziantep, Turkey) and an autoanalyzer (Abbott Architect C16000, Abbott Diagnostics Laboratories), based on automatic colorimetric methods described by Erel et al. [20] TAS results are offered as micromolar Trolox equivalent per liter $(\mu \mathrm{mol}$ Trolox equiv/L). TOS results are shown as micromolar hydrogen peroxide per liter $\left(\mu \mathrm{mol} \mathrm{H}_{2} \mathrm{O}_{2}\right.$ equiv/L). Malondialdehyde (MDA) levels were detected by spectrophotometric measurement of the color produced between the reaction of thiobarbituric acid and MDA [21].

Serum levels of interleukin 1 (IL-1), interleukin 6 (IL-6), interleukin 10 (IL-10) and tumor necrosis factor- $\alpha$ (TNF- $\alpha$ ) were measured using the ELISA method, in accordance with the manufacturer's instructions. Biochemical parameter results were recorded. Tissues weighing $0.13-0.25 \mathrm{~g}$ were washed with saline water, then dried using blotting paper. Next, the tissues were placed in Eppendorf tubes and 
stored at $-80^{\circ} \mathrm{C}$ until use. Before biochemical analysis, the samples were brought to $-20^{\circ} \mathrm{C}$, warmed to $+4^{\circ} \mathrm{C}$, and gradually thawed. Tissues were homogenized in phosphate-buffered saline ( $\mathrm{pH}$ 7.4) on ice using a WiseTis Homogenizer. Tissues were centrifuged at 3,000 rpm for $10 \mathrm{~min}$ in a Nüve $\mathrm{T}^{\mathrm{TM}}$ centrifuge and the supernatant was collected. Tissues were prepared for ELISA and washed in a BioTek ELX50 microplate washer; ELISA results were recorded at $450 \mathrm{~nm}$ (Triturus GRI FOLS ${ }^{\mathrm{TM}}$ ). TAS, TOS, MDA, TNF- $\alpha$, IL-1, IL-6 and IL-10 levels were evaluated in liver tissues. The optical density values and standard (std) concentration obtained from the standard solution were used to plot the distributions of each inflammatory cytokine in Excel software. The formula calculated based on the curve was used to calculate the concentrations of the samples by means of their optical densities.

\section{Statistical analysis}

Statistical analyses were computed by SPSS Statistics, version 24.0; scores were shown as mean \pm standard deviation (SD). KruskalWallis and Mann-Whitney U tests were used for differences between group comparisons. When a $p$ value was less than 0.05 , the differences were considered as statistically significant.

\section{Results}

\section{Histopathological findings}

Ductal proliferation, inflammation, necrosis, and fibrosis were found to be significantly increased in the liver tissues of all groups with biliary obstruction compared to the Group 1 . Although necrosis and ductal proliferation were improved in Group 3, no statistically significant difference was observed when compared with Group 2. However, when Groups 2 (Figures 1A, B and C) and 3 (Figure 2A, B and C) were compared, fibrosis and inflammation levels were statistically significantly lower in group 3 $(\mathrm{p}<0.05$; Table 1$)$.

\section{Liver function tests (LFTs)}

All LFTs values studied in groups with biliary duct obstruction (Group 2 and Group 3) were significantly higher $(\mathrm{p}<0.05)$ compared to group 1 values. When group 2 and group 3 were compared; all LFTs except DBIL were significantly lower $(\mathrm{p}<0.05)$ in group 3 (Table 2) than group 2.

\section{Liver interleukin levels and oxidative stress parameters}

The liver IL-6, TOS, MDA, and TNF- $\alpha$ levels were significantly $(\mathrm{p}<0.05)$ increased in the biliary obstruction groups (Group 2 and group $3)$ compared to the Group 1. When groups 2 and 3 were compared, IL-6, IL-1, TOS, MDA, and TNF- $\alpha$ levels were significantly lower $(\mathrm{p}<$ 0.05 ) in the group 2, whereas IL-10 and TAS values were significantly higher in group 3 . In addition, mean TAS values were significantly higher in group 3 than in group 1 (Table 3 ).

\section{Blood interleukin and oxidative stress parameters}

Significant increases in TNF- $\alpha$ levels, MDA, IL-6, IL-1, and TOS, as well as a significant decrease in IL-10 levels, were followed in

Table 1. Comparison of liver histopathological evaluation of the groups.

\begin{tabular}{|l|l|l|l|l|}
\hline Groups & Ductal proliferation & Fibrosis & Inflammation & Necrosis \\
\hline G $1(\mathbf{n}=\mathbf{1 0})$ & $0,01 \pm 0,01$ & $0,01 \pm 0,01$ & $0,42 \pm 0,20$ & $0,42 \pm 0,29$ \\
\hline G $2(\mathbf{n}=\mathbf{1 0})$ & $2,28 \pm 0,28^{*}$ & $1,28 \pm 0,18^{*}$ & $1,71 \pm 0,18^{*}$ & $4,14 \pm 0,73^{*}$ \\
\hline G 3 (n=10) & $1,57 \pm 0,29^{*}$ & $0,42 \pm 0,20^{* \mathbf{\Lambda}}$ & $0,85 \pm 0,14^{* \mathbf{\Lambda}}$ & $2,57 \pm 0,36^{*}$ \\
\hline
\end{tabular}

Values are expressed as mean \pm SD. G1: Sham-operated group. G2: Control group. G3: GA group. * Group 1 vs Group 2 and Group $3 p<0.05 ; \wedge$ Group 2 vs Group $3 p<0.05$. 
group 2, compared with group $1(\mathrm{p}<0.05)$. However, in group 3, the IL-6, IL-1, TOS, MDA, and TNF- $\alpha$ levels significantly decreased, while IL-10 and TAS levels significantly increased $(p<0.05)$, compared with group 2. In addition, no significant differences were followed between groups 1 and 3 for all parameters, except MDA and TNF$\alpha$; however, MDA and TNF- $\alpha$ values were higher in group 3 than in group $1(\mathrm{p}<0.05$; Table 4).

\section{Discussion}

Cholestasis, known as disruption of biliary flow, leads to the collection of bile acids and other toxins in the liver; this causes hepatotoxicity, thus damaging intracellular compartments and cell membranes. The main hypothesis regarding the mechanism of liver damage due to liver cholestasis is that hepatocellular apoptosis increases due to bile acid accumulation in hepatocytes and blood [22]. An alternative hypothesis is that excess

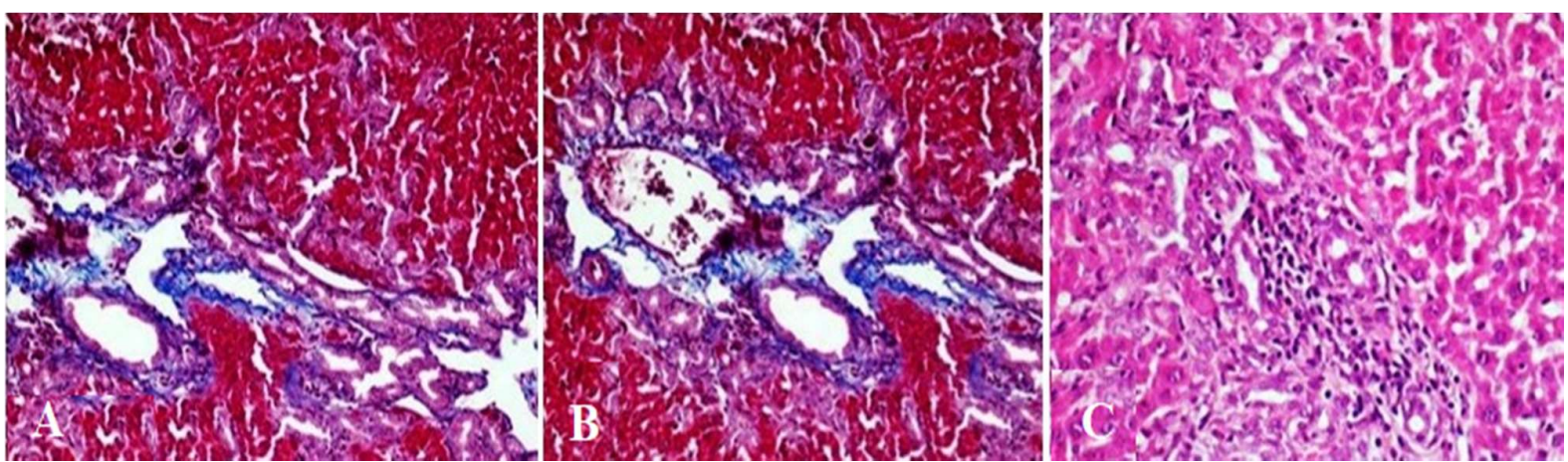

Figure 1. In control group (Group 2), fibrous expansion of some portal areas (A, B) (Masson's trichrome, x20); moderate portal inflammation (C) (Masson's trichrome, x20).
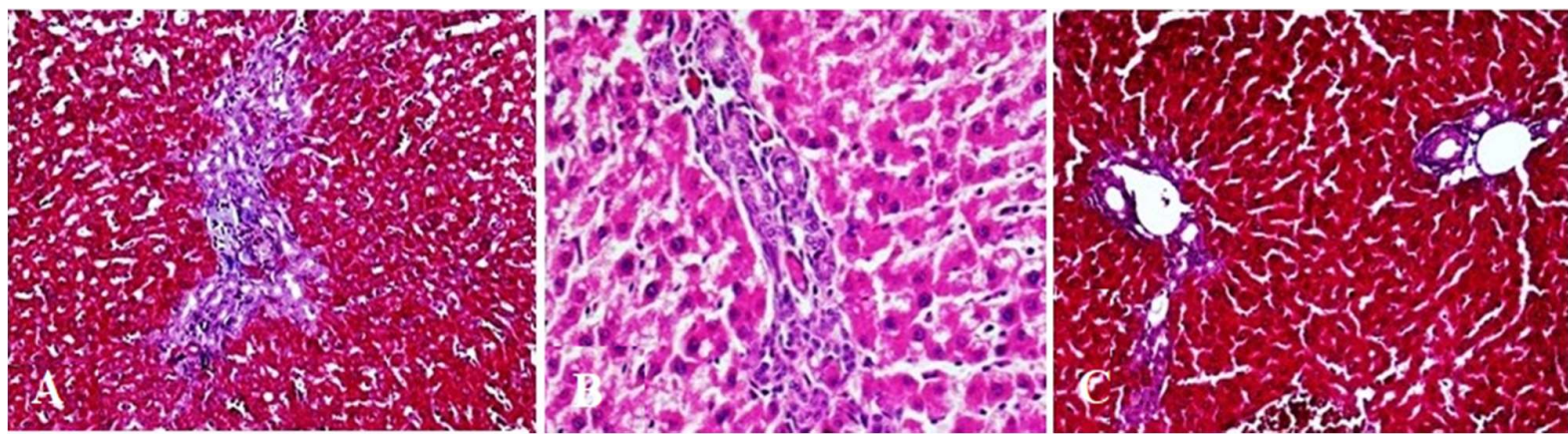

Figure 2. In gallic acid treated group (Group 3), mild portal fibrosis (A) (Masson's trichrome, x20); no portal inflammation (B) (Masson's trichrome, x20); no portal fibrosis (C) (Masson's trichrome, x20).

Table 2. Comparison of liver function tests of the groups.

\begin{tabular}{|l|l|l|l|l|l|l|}
\hline Groups & LDH & GGT & AST & ALT & TBIL & DBIL \\
\hline G 1 (n=10) & $261 \pm 29,1$ & $10,1 \pm 1,2$ & $105 \pm 6,8$ & $39 \pm 2,9$ & $0,27 \pm 0,10$ & $0,06 \pm 0,01$ \\
\hline G $2(\mathbf{n = 1 0})$ & $1426 \pm 82,3^{*}$ & $74,7 \pm 3,3^{*}$ & $883 \pm 104^{*}$ & $218 \pm 28^{*}$ & $11,9 \pm 1,20^{*}$ & $7,58 \pm 0,91^{*}$ \\
\hline G 3 (n=10) & $768 \pm 50,4^{* \mathbf{\Lambda}}$ & $38 \pm 1^{* \mathbf{\Lambda}}$ & $407 \pm 40^{* \mathbf{\Lambda}}$ & $102 \pm 12^{* \mathbf{\Lambda}}$ & $7,30 \pm 0,54^{* \mathbf{\Lambda}}$ & $6,15 \pm 0,78^{*}$ \\
\hline
\end{tabular}

Values are expressed as mean \pm SD. G1: Sham-operated group. G2: Control group. G3: GA group. * Group 1 vs Group

2 and Group 3 p<0.05; $\triangle$ Group 2 vs Group 3 p<0.05. ALT: Alanine aminotransferase; AST: Aspartate aminotransferase; DBIL: Direct bilirubin; GGT: Gamma-glutamyl transferase; LDH: Lactate dehydrogenase; TBIL: Total bilirubin. 
Table 3. Comparison of liver interleukin and oxidative stress parameters.

\begin{tabular}{|l|l|l|l|l|l|l|l|}
\hline Groups & IL- 6 & IL-1 & IL-10 & TAS & TOS & MDA & TNF- $\boldsymbol{A}$ \\
\hline G 1 (n=10) & $65 \pm 6,5$ & $1282 \pm 118$ & $151 \pm 20$ & $0,81 \pm 0,04$ & $15,2 \pm 1,01$ & $12,3 \pm 0,94$ & $250 \pm 115$ \\
\hline G 2 (n=10) & $182 \pm 11,5^{*}$ & $2034 \pm 175^{*}$ & $105 \pm 15,1$ & $0,87 \pm 0,04$ & $34,8 \pm 2,1^{*}$ & $42,2 \pm 1,67 *$ & $350 \pm 120^{*}$ \\
\hline G 3 (n=10) & $111 \pm 8,5^{* \mathbf{\Lambda}}$ & $1273 \pm 94^{\mathbf{\Lambda}}$ & $217 \pm 26^{\mathbf{\Delta}}$ & $1,48 \pm 0,86^{\mathbf{4}}$ & $23,8 \pm 2,1^{* \mathbf{\Lambda}}$ & $22,8 \pm 2,38^{* \mathbf{\Lambda}}$ & $275 \pm 120^{* \mathbf{\Lambda}}$ \\
\hline
\end{tabular}

Values are expressed as mean $\pm S D$. G1: Sham-operated group. G2: Control group. G3: GA group. * Group 1 vs Group

2 and Group 3 p<0.05; \ Group 2 vs Group $3 p<0.05$; ${ }^{*}$ Group 1 vs Group $3 p<0.05$. IL, interleukin; MDA:

Malondialdehyde; TAS: Total antioxidant status; TNF- $\alpha$ : Tumor necrosis factor- $\alpha$; TOS: Total oxidant status.

Table 4. Comparison of serum interleukin and oxidative stress parameters.

\begin{tabular}{|c|c|c|c|c|c|c|c|}
\hline Groups & IL- 6 & IL-1 & IL-10 & TAS & TOS & MDA & TNF- $\alpha$ \\
\hline G 1 ( & $83 \pm 22 *$ & $1348 \pm 173^{*}$ & $202,1 \pm 41 *$ & $1,1 \pm 0,2 *$ & $18,7 \pm 6^{*}$ & $14 \pm 3,8^{* *}$ & $251 \pm 9 * *$ \\
\hline G $2(n=10)$ & $170,5 \pm 50^{\Lambda *}$ & $1783 \pm 497^{\star} *$ & $106 \pm 28^{\wedge} *$ & $0,8 \pm 0,1^{\wedge} *$ & $28 \pm 3,8^{\boldsymbol{\Delta}} *$ & $37 \pm 9^{\wedge} *$ & $338 \pm 35^{\wedge} *$ \\
\hline G $3(n=10)$ & $107 \pm 42^{\wedge}$ & $1277 \pm 233^{\mathbf{\Lambda}}$ & $176 \pm 47^{\mathbf{\Lambda}}$ & $1,4 \pm 0,3^{\wedge}$ & $21 \pm 6^{\mathbf{\Lambda}}$ & $21,6 \pm 7^{\Perp} ¥$ & $275 \pm 15^{\Delta ¥}$ \\
\hline
\end{tabular}

Values are expressed as mean \pm SD. G1: Sham-operated group. G2: Control group. G3: GA group. * G1, G2 vs G3 $p<0.05 ;{ }^{\wedge} G 2$ vs $G 3 P<0.05 ;{ }^{*} G 1$ vs $G 3 P<0.05$. IL: Interleukin; MDA: Malondialdehyde; TAS: Total antioxidant status; TNF- $\alpha$ : Tumor necrosis factor- $\alpha$; TOS: Total oxidant status.

accumulation of bile acids occurs in the blood and liver (sterile inflammation); many studies have been performed regarding this issue. The toxic build-up of bile acids is an important mechanism for cell toxicity, including oxidative stress, apoptosis, and fibrosis [23].

Neutrophils are recruited to the area of injury approximately six hours after bile duct ligation; they peak at 48-72 hours, when liver damage is most commonly observed [24]. Neutrophil accumulation is directly associated with injury or inflammation. Neutrophils can be especially toxic to leukocytes when superoxides are generated by potent oxidant hypochlorous acid and nicotinamide adenine dinucleotide phosphate oxidase is generated by myeloperoxidase. This shows that hypochlorous acid can cause intracellular oxidant stress in hepatocytes and consequently cause cell damage [25].
Histopathological findings have been observed in the liver following bile duct ligation in various studies. In the study by Hongwei et al., livers with bile duct ligation showed elevated bile duct proliferation, parenchymal necrosis, and inflammatory cell infiltration. When Hongwei et al. treated the rats with retinoic acid and ursodeoxycholic acid, the pathological changes were reduced and liver tissues were restored to normal structure [26].

In another study by Zong et al., less collagen deposition was observed in the group subjected to both bile duct ligation and treatment with ethyl pyruvate. In addition, the liver fibrosis score was lower in rats treated with ethyl pyruvate. Thus, Zong et al. concluded that treatment with ethyl pyruvate delayed liver fibrosis [27].

In the present study, inflammation and cellular necrosis were observed by means of 
histological analysis in both bile duct ligation groups (groups 2 and 3). This was an indicator of neutrophil collection after bile duct ligation. Furthermore, histological examination revealed a reduction in the parameters associated with liver fibrosis and inflammation in the group 3, which confirmed the above-mentioned morphological results.

Gamma-glutamyl transferase, bilirubin, and LFTs (e.g., AST, ALT, and lactate dehydrogenase) are particularly sensitive determinants of liver damage and dysfunction in membrane transport, as well as in cell and mitochondrial membrane properties [28].

Zong et al. reported that the bile duct ligation group had significantly higher serum AST and ALT levels, compared with the sham group. Ethyl pyruvate treatment significantly reduced ALT and AST levels, compared with the bile duct ligation group [27].

In the study by Xie et al., the serum ALT, AST, and total bilirubin levels were significantly lower in the bile duct ligation + dexmedetomidine group than in the bile duct ligation group [29].

In the present study, significant increases in LFTs were observed in the biliary obstruction groups (Groups 2 and 3). However, a decrease in LFTs (except direct bilirubin) in the group 3 compared to Group 2 indicated that GA inhibits or reduces OC-induced liver membrane damage due to its antioxidant properties; this findings is consistent with the results of other studies [30,31].

The anti-inflammatory and antioxidant properties of GA may be associated with elevated liver superoxide dismutase and liver catalase activities, liver TNF- $\alpha$ expression, liver MDA, and serum protein carbonyl reduction [31]. MDA is an indicator of lipid peroxidation and a commonly used marker of oxidative stress [14]. In the study by Cao et al., elevated MDA was observed in liver tissue after bile duct ligation; rats in the GA-treated group showed reduced MDA levels [32]. In the study by Demirbilek et al., the MDA level was significantly increased in rats with bile duct ligation. However, decreased MDA levels were observed in rats treated with sulfasalazine and pyrrolidine dithiocarbomate [33]. In the present study, due to bile duct ligation-induced liver injury, MDA levels in liver and serum were significantly higher in Group 2 than in Group 1. Conversely, treatment with GA led to lower MDA levels in liver tissue and serum, which is consistent with the previously reported findings [34]. These effects may be, at least in part, due to the antioxidant properties of GA.

TAS and TOS measurements are valuable to evaluate oxidative stress. The TOS is an important indicator of oxidative stress [35], while TAS functions to preserve tissue integrity from oxidative injury. The TAS is a precise indicator of antioxidant level [36]. In the study by Coban et al., the TOS level increased and the TAS level decreased in rats in the bile duct ligation model; conversely, the TOS level decreased and the TAS level increased in the treatment group [37]. Similar results were observed in other experimental injury models [38]. In the present study, the TOS level was significantly higher in the Group 2 than in Group 1. In the Group 3, a significant decrease in TOS and a significant increase in TAS levels were observed $(p<0.05)$, presumably due to the antioxidant properties of GA.

IL-1 and IL-6 play crucial roles in inflammatory events and immunity $[39,40]$. In the liver, TNF- $\alpha$ production induces apoptosis and necrosis of hepatocytes, leading to liver inflammation [41]. In a study by Xie et al., dexmedetomidine treatment decreased serum IL-6 and TNF- $\alpha$ proinflammatory cytokine levels, compared with the bile duct ligation 
group [29]. In a study by Pan et al., following bile duct ligation, IL-1 levels were improved in the treated rats [42].

In the present study, significantly higher serum TNF- $\alpha$, IL-1, and IL-6 levels were observed in Group 2 ( $\mathrm{p}<0.05)$. However, Group 3 showed significantly lower serum TNF- $\alpha$, IL-1 and IL6 levels, compared with Group $2(\mathrm{p}<0.05)$. Thus, the observed reductions in TNF- $\alpha$, IL-1, and IL-6 serum levels confirm that GA has hepatoprotective effects via its antiinflammatory properties.

IL-10 is a Th2-type cytokine that exerts inflammatory effects by inhibiting the production of pro-inflammatory cytokines (e.g., TNF-alpha, IL-1, IL-6, and IL-8). In addition, IL-10 plays a role in regulating monocyte production of soluble TNF- $\alpha$ and IL-1 receptor antagonist [43]. In the present study, IL-10 levels were lower in the group 2 than in the group 1, which is consistent with previously reported results. However, IL-10 levels were increased in Group 3 compared to Group 2 and Group $1(\mathrm{p}<0.05)$.

\section{Conclusion}

Findings of this research indicate that GA may be effective against $\mathrm{OC}$-induced liver damage in a rat model. We presume that the beneficial effects of GA are closely associated with its antioxidant and anti-inflammatory activities. Therefore, we think that using GA can save us time before resorting to a surgical method. However, further studies are needed to investigate the optimal dosage and duration of application.

\section{Abbreviations}

GA: Gallic acid

OC: Obstructive cholestasis

LFTs: Liver function tests

ALT: Alanine aminotransferase

AST: Aspartate aminotransferase
TBIL: Total bilirubin

DBIL: Direct bilirubin

LDH: Lactate dehydrogenase

GGT: Gamma-glutamyl transferase

ROS: Reactive oxygen species

ELISA: Enzyme-linked immunosorbent assays

TAS: Total antioxidant status

TOS: Total oxidant status

MDA: Malondialdehyde

IL: Interleukin

TNF- $\alpha$ : Tumor necrosis factor- $\alpha$

EF: Ethyl acetate fraction

Funding: This study was supported by a grant from the Scientific Research and Project Coordinator (DUBAP, 14-TF-149) at Dicle University, Diyarbakir, Turkey.

Conflict of Interest: The authors declare that they have no conflict of interest.

Ethical statement: The study were confirmed by the Dicle University Animal Experiments Local Ethics Committee (DUHADEK) (Decision No: 14-TF-149)

ORCID iD of the author(s)

Erol Basuguy / 0000-0002-9217-423X

Mehmet Hanifi Okur / 0000-0002-6720-1515

Serkan Arslan / 0000-0002-5879-4478

Hikmet Zeytun / 0000-0002-7159-952X

Gulay Aydogdu / 0000-0002-3784-7622

Aysun Ekinci / 0000-0002-0547-4139

Copyrights: (C) 2021@author (s).

This is an open access article distributed under the terms of the Creative Commons Attribution License (CC BY 4.0), which permits unrestricted use, distribution, and reproduction in any medium, provided the original author(s) and source are credited and that the original publication in this journal is cited, in accordance with accepted academic practice. 
No use, distribution or reproduction is permitted which does not comply with these terms.

\section{References}

[1]Gondal B, Aronsohn A. A Systematic Approach to Patients with Jaundice.Semin Intervent Radiol. 2016;33(4):253-58. Review.

[2]Emerick KM, Whitington PF. Molecular basis of neonatal cholestasis. Pediatr Clin North Am. 2002;49(1):221-35.

[3]Li Y, Tang R, Leung PSC, et al. Bile acids and intestinal microbiota in autoimmune cholestatic liver diseases. Autoimmun Rev. 2017;16(9):885-96.

[4]Pauli-Magnus C, Meier PJ. Hepatocellular transporters and cholestasis. J Clin Gastroenterol. 2005;39(4 Suppl 2):S103-10.

[5]Navaneethan U, Jayanthi V, Mohan P. Pathogenesis of cholangitis in obstructive jaundice-revisited. Minerva Gastroenterol Dietol. 2011;57(1):97-104.

[6]Trauner M, Wagner M, Fickert P, et al. Molecular regulation of hepatobiliary transport systems: clinical implications for understanding and treating cholestasis. J Clin Gastroenterol. 2005;39(4 Suppl 2):S111-24.

[7]Addley J, Mitchell RM. Advances in the investigation of obstructive jaundice. Curr Gastroenterol Rep. 2012;14(6):511-19.

[8]Zhu L, Wang L, Cao F, et al. Modulation of transport and metabolism of bile acids and bilirubin by chlorogenic acid against hepatotoxicity and cholestasis in bile duct ligation rats: involvement of SIRT1mediated deacetylation of FXR and PGC- $1 \alpha$. J Hepatobiliary Pancreat Sci. 2018;25(3):195-205.

[9]Eslami AC, Pasanphan W, Wagner BA, et al. Free radicals produced by the oxidation of gallic acid: An electron paramagnetic resonance study. Chem Cent J. 2010;4:15.

[10] Chen Y, Zhou G, Ma B, et al. Active Constituent in the Ethyl Acetate Extract Fraction of Terminalia bellirica Fruit Exhibits Antioxidation, Antifibrosis, and Proapoptosis Capabilities In Vitro. Oxid Med Cell Longev. 2019;2019:5176090.

[11]Zhou ZH, Liu MZ, Wang MH, et al. A new ellagic acid derivative from Polygonum runcinatum. Nat Prod Res. 2015;29(9):79599.

[12] Nabavi SF, Habtemariam S, Jafari M,et al. Protective role of gallic acid on sodium fluoride induced oxidative stress in rat brain. Bull Environ Contam Toxicol. 2012;89(1):73-77.

[13] Chao WW, Chen SJ, Peng HC, et al. Antioxidant Activity of Graptopetalum paraguayense E. Walther Leaf Extract Counteracts Oxidative Stress Induced by Ethanol and Carbon Tetrachloride CoInduced Hepatotoxicity in Rats. Antioxidants (Basel). 2019;8(8). pii: E251.

[14] Kim YJ. Antimelanogenic and antioxidant properties of gallic acid. Biol Pharm Bull. 2007;30(6):1052-55.

[15] Priscilla DH, Prince PS. Cardioprotective effect of gallic acid on cardiac troponin-T, cardiac marker enzymes, lipid peroxidation products and antioxidants in experimentally induced myocardial infarction in Wistar rats. Chem Biol Interact. 2009;179(2-3):118-24.

[16]Criado M, Flores O, Ortíz MC, et al. Elevated glomerular and blood mononuclear lymphocyte nitric oxide production in rats with chronic bile duct ligation: role of inducible nitric oxide synthase activation. Hepatology. 1997;26(2):268-76.

[17] Yu Z, Song F, Jin YC, et al. Comparative Pharmacokinetics of Gallic Acid After Oral Administration of Gallic Acid Monohydrate 
in Normal and Isoproterenol-Induced Myocardial Infarcted Rats. Front Pharmacol. 20186;9:328.

[18] Ishak K, Baptista A, Bianchi L, et al. Histological grading and staging of chronic hepatitis. J Hepatol. 1995;22(6):696-99.

[19] Ara C, Kirimlioglu H, Karabulut AB, et al. Protective effect of resveratrol against oxidative stress in cholestasis. J Surg Res. 2005;127(2):112-17.

[20]Erel O. A novel automated method to measure total antioxidant response against potent free radical reactions. Clin Biochem. 2004;37(2):112-19.

[21]Esterbauer H, Cheeseman KH. Determination of aldehydic lipid peroxidation products: malonaldehyde and 4-hydroxynonenal. Methods Enzymol. 1990;186:407-21.

[22] Maillette de Buy Wenniger L, Beuers U. Bile salts and cholestasis. Dig Liver Dis. 2010;42(6):409-18.

[23]Yang H, Ramani $K$, Xia $M$, et al. Dysregulation of glutathione synthesis during cholestasis in mice: molecular mechanisms and therapeutic implications. Hepatology. 2009;49(6):1982-91.

[24] Georgiev P, Jochum W, Heinrich S, et al. Characterization of time-related changes after experimental bile duct ligation. $\mathrm{Br} \mathrm{J}$ Surg. 2008;95(5):646-56.

[25] Woolbright BL, Jaeschke H. Novel insight into mechanisms of cholestatic liver injury. World J Gastroenterol. 2012;18(36):498593.

[26] He H, Mennone A, Boyer JL, et al. Combination of retinoic acid and ursodeoxycholic acid attenuates liver injury in bile duct-ligated rats and human hepatic cells. Hepatology. 2011;53(2):548-57.

[27]Zong Y, Zhang M, Li S, et al. Effects of Ethyl Pyruvate on Bile Duct Ligation-
Induced Liver Fibrosis by Regulating Nrf2 Pathway and Proinflammatory Cytokines in Rats. Gastroenterol Res Pract. 2019;2019:2969802.

[28]Feldman M, Friedman LS, Brandt LJ. Sleisenger and Fordtran's Gastrointestinal and Liver Disease (9th Eds): Philadelphia, PA: Saunders Elsevier, 2010.

[29]Xie Y, Guo C, Liu Y, et al. Dexmedetomidine activates the PI3K/Akt pathway to inhibit hepatocyte apoptosis in rats with obstructive jaundice. Exp Ther Med. 2019;18(6):4461-66.

[30] Inkielewicz-Stępniak I. Impact of fluoxetine on liver damage in rats. Pharmacol Rep. 2011;63(2):441-47.

[31]Karimi-Khouzani O, Heidarian E, Amini SA. Anti-inflammatory and ameliorative effects of gallic acid on fluoxetine-induced oxidative stress and liver damage in rats. Pharmacol Rep. 2017;69(4):830-35.

[32]Cao F, Liu P, Zhang X, et al. Shenqi Fuzheng Injection impairs bile duct ligationinduced cholestatic liver injury in vivo. Biosci Rep. 2019;39(1). pii: BSR20180787.

[33] Demirbilek S, Akin M, Gürünlüoğlu K, et al. The NF-kappa B inhibitors attenuate hepatic injury in bile duct ligated rats. Pediatr Surg Int. 2006;22(8):655-63.

[34] Okur MH, Zeytun H, Basuguy E, et al. Protective effects of cordycepin on the histopathological changes and oxidative stress induced by hepatic ischemia/reperfusion in rats. Exp Biomed Res. 2018; 1(1):17-24.

[35] Aycicek A, Ipek A. Maternal active or passive smoking causes oxidative stress in cord blood. Eur J Pediatr. 2008;167(1):8185.

[36] Akcilar R, Akcilar A, Savran B, et al. Effects of ukrain in rats with intestinal ischemia and reperfusion. J Surg Res. 2015;195(1):67-73. 
[37] Coban S, Yildiz F, Terzi A, et al. The effects of Nigella sativa on bile duct ligation induced-liver injury in rats. Cell Biochem Funct. 2010;28(1):83-88.

[38]Zeren S, Bayhan Z, Koçak C, et al. Antioxidant Effect of Ukrain Versus NAcetylcysteine Against Acute Biliary Pancreatitis in An Experimental Rat Model. J Invest Surg. 2017;30(2):116-24.

[39] Nishimoto N, Kishimoto T. Interleukin 6: from bench to bedside. Nat Clin Pract Rheumatol. 2006;2(11):619-26.

[40] Stylianou E, Saklatvala J. Interleukin-1. Int J Biochem Cell Biol. 1998;30(10):1075-79.

[41] Tracey KJ. The inflammatory reflex. Nature. 2002;420(6917):853-59.

[42] Pan PH, Lin SY, Wang YY, et al. Protective effects of rutin on liver injury induced by biliary obstruction in rats. Free Radic Biol Med. 2014;73:106-16.

[43] Cassatella MA, Meda L, Bonora S, et al. Interleukin 10 (IL-10) inhibits the release of proinflammatory cytokines from human polymorphonuclear leukocytes. Evidence for an autocrine role of tumor necrosis factor and IL-1 beta in mediating the production of IL-8 triggered by lipopolysaccharide. J Exp Med. 1993;178(6):2207-11. 